\section{Validade da autopercepção da presença de cárie dentária como teste diagnóstico e fatores associados entre adultos}

\author{
Validity of self-perceived dental caries as a \\ diagnostic test and associated factors \\ in adults
}

\section{Validez de la autopercepción de la presencia de caries dental como test diagnóstico y factores asociados entre adultos}

\section{Resumo}

Objetivou-se investigar a validade da autopercepção da presença da cárie dentária e fatores associados, mediante estudo com 795 adultos (35-44 anos). A variável dependente foi autopercepção da presença da cárie dentária, as independentes reunidas em blocos. Três modelos logísticos foram conduzidos: (1) todos os adultos; (2) adultos com lesões de cárie normativa; e (3) adultos sem tais lesões. A autopercepção da presença da cárie dentária apresentou sensibilidade de 77,7\%, especificidade de 58\%, acurácia de 65\%, valor preditivo positivo de $52 \%$ e valor preditivo negativo de $81 \%$. No Modelo 1, a autopercepção da presença da cárie dentária foi associada ao tempo de uso dos serviços odontológicos, acesso à informação, uso de fio dental, lesões normativas, percepção da necessidade de tratamento, dor, insatisfação com a saúde bucal e geral. No Modelo 2, a autopercepção da presença da cárie dentária foi associada ao tempo de uso dos serviços odontológicos, percepção da necessidade de tratamento e insatisfação com a saúde bucal e geral. No Modelo 3, autopercepção da presença da cárie dentária foi associada ao tempo de uso dos serviços odontológicos, acesso à informação, percepção da necessidade de tratamento, insatisfação com a saúde bucal e uso de fio dental. A autopercepção da presença da cárie dentária mostrou utilidade limitada como método de diagnóstico.

Autoimagem; Cárie Dentária; Saúde Bucal
Desirée Sant'Ana Haikal 1

Luana Leal Roberto 1

Andréa Maria Eleutério de Barros Lima Martins 1

Alfredo Maurício Batista de Paula 1

Efigênia Ferreira e Ferreira 2

doi: 10.1590/0102-311X00053716

\section{Correspondência}

D. S. Haikal

Universidade Estadual de Montes Claros.

Av. Cula Mangabeira 562, Montes Claros, MG 39401-002,

Brasil.

luleal15@yahoo.com.br

1 Universidade Estadual de Montes Claros,

Montes Claros, Brasil.

2 Universidade Federal de Minas Gerais, Belo Horizonte, Brasil. 


\section{Introdução}

A autopercepção ou "autoavaliação" em saúde bucal é a capacidade individual e subjetiva de perceber e avaliar a própria saúde bucal. Baseia-se em informações e conhecimentos disponíveis, mediados por experiências prévias, contexto social, cultural e histórico de cada indivíduo ${ }^{1,2}$. O estudo da autopercepção tem recebido atenção crescente na área da saúde. Já foi observado que o conceito é útil para gestão/planejamentos dos serviços de saúde, na prevenção, prática clínica e em pesquisas 3,4 . Assim, a autopercepção da saúde bucal pode ajudar na avaliação, organização e financiamento dos serviços de saúde, nas intervenções de promoção de saúde, na avaliação das necessidades de tratamento, bem como na identificação de indivíduos que potencialmente mais se beneficiariam com o tratamento odontológico 1,3,4,5,6. Além disso, já foi confirmado que ela influencia a qualidade de vida dos indivíduos 7, a aceitação dos planos de tratamento 6 , a frequência de procura por atendimento odontológico 1,8 e pode captar qual a consciência que os indivíduos têm de sua própria condição bucal, consciência que influencia seus comportamentos em saúde 9 .

Estudos referentes à autopercepção da saúde bucal podem oferecer oportunidade de aferição da condição bucal e levam em conta o que é aceitável por parte dos indivíduos uma vez que o exame clínico aumenta os custos dos inquéritos epidemiológicos em saúde bucal 10,11. Além da limitação do custo, há que se considerar também que mesmo os índices puramente clínicos/normativos não estão livres da subjetividade inerente ao olhar do examinador. Esses índices são definidos por especialistas humanos, que estabelecem a presença e a severidade das condições patológicas, baseado no conhecimento disponível 2. Dessa forma, algum grau de variação ou erro de diagnóstico pode ocorrer intra ou interexaminadores 12 . Fatores físicos e psicológicos tais como fadiga, flutuações no interesse pelo estudo, dificuldade na tomada de decisões e variações na acuidade visual e no senso tátil podem afetar o julgamento dos examinadores em algumas ocasiões e em diferentes estágios, afetando a consistência das observações clínicas 13 .

A cárie dentária representa um importante problema de saúde pública no Brasil 14 e no mundo 15. Representa o problema de saúde bucal mais prevalente; próximo de $99 \%$ dos adultos brasileiros já apresentaram alguma experiência de cárie 16. Apesar disso, são escassos os estudos que tenham avaliado fatores associados à autopercepção da cárie dentária pelos indivíduos 11, sejam eles portadores ou não de lesões normativas. O entendimento dos fatores que contribuem para uma percepção adequada da presença da cárie dentária é importante, tendo em vista sua grande utilidade potencial como um indicador de saúde bucal 17 .

Como os adultos constituem a maioria da população, representam o estrato populacional predominantemente ativo do ponto de vista econômico, além de poderem influenciar de forma decisiva o comportamento de seus dependentes 18 , este estudo objetivou identificar os fatores associados à autopercepção da presença da cárie dentária entre adultos, considerando os portadores e os não portadores de lesões cariosa conforme diagnósticos normativos.

\section{Metodologia}

Estudo transversal conduzido em uma amostra representativa de adultos (35-44 anos) 12 de um município de grande porte, localizado no Estado de Minas Gerais, Brasil. A metodologia adotada foi baseada no levantamento das Condições de Saúde Bucal da População Brasileira (Projeto SBBrasil) 19 e nas preconizações da Organização Mundial da Saúde (OMS) 12. O estudo foi previamente aprovado por Comitê de Ética em Pesquisa (parecer número no 318/06 - Unimontes).

Foi adotada amostragem complexa, probabilística por conglomerados em dois estágios (setores censitários e quadras), garantindo proporcionalidade por sexo. Os cálculos indicaram a necessidade de examinar 762 indivíduos adultos, considerando a ocorrência dos eventos ou doenças em 50\%, intervalo de $95 \%$ de confiança, erro tolerável de 5\%, deff igual a 2,0 e taxa de não resposta de $20 \%$.

Os domicílios situados nas quadras sorteadas foram visitados, e os adultos convidados a participar. Após a assinatura do termo de consentimento livre e esclarecido, foram conduzidas entrevistas e exames intrabucais realizados por cirurgiões-dentistas treinados e calibrados, que alcançaram concordância kappa satisfatória 20, acompanhados de anotadores/digitadores (acadêmicos) treinados. 
Os exames foram conduzidos sob luz natural, com auxílio de espelho e adotando todos os códigos/ critérios propostos pela OMS 12 .

Os dados foram registrados em computadores de mão, utilizando um programa criado especificamente para esse fim que permitiu construção simultânea do banco de dados. A análise dos dados foi conduzida com emprego do programa estatístico PASW (SPSS Inc., Chicago, Estados Unidos).

A variável dependente estudada foi a autopercepção da presença da cárie dentária, obtida por meio da seguinte pergunta da entrevista: "Você acha que tem cárie dentária?" (sim/não). As variáveis independentes foram reunidas em cinco grupos: sociodemográficas, utilização de serviços odontológicos, condições normativas de saúde bucal, autopercepção da saúde e hábitos.

As varáveis sociodemográficas foram: idade (35-39 anos, 40-44 anos); sexo (feminino, masculino); cor da pele autodeclarada (branco, não branco); local de residência (zona urbana, zona rural); renda per capita ( $>\mathrm{R} \$ 200,00, \leq \mathrm{R} \$ 200,00$ ); escolaridade em anos ( $>8$ anos, $\leq 8$ anos, analfabeto-sem escolaridade); união estável (sim, não); posse de automóvel (sim, não).

As variáveis da utilização dos serviços odontológicos foram: tipo de serviço odontológico utilizado (privado, supletivo ou outro/público); tempo do último uso do serviço odontológico ( $\leq 1$ ano, $>1$ ano); motivo do uso (rotina-manutenção, problemas odontológicos); recebeu informação sobre como evitar problemas bucais (sim, não).

As variáveis relativas às condições normativas de saúde bucal foram: número de dentes presentes $(\geq 20,<20)$; cárie dentária normativa (ausente, presente); doença periodontal (ausente, presente); lesão na mucosa (ausente, presente); necessidade de tratamento de urgência (não, sim). A doença periodontal foi considerada presente naqueles indivíduos que apresentaram, num mesmo sextante, bolsa periodontal $\geq 4 \mathrm{~mm}$ (índice periodontal comunitário - CPI) e perda de inserção $\geq 4 \mathrm{~mm}$ (índice de perda de inserção periodontal - PIP) 21.

O grupo das variáveis da autopercepção da saúde foi constituído por: autopercepção da necessidade de tratamento odontológico (não, sim); autopercepção da aparência (excelente-boa, regular-ruimpéssima); autopercepção da mastigação (excelente-boa, regular-ruim-péssima); autopercepção da fala (excelente-boa, regular-ruim-péssima); autopercepção do relacionamento social (não afeta, afeta); autopercepção do paladar (excelente-boa, regular-ruim-péssima); autopercepção de mau hálito (não percebido, percebido); autopercepção da saúde bucal (satisfeito, insatisfeito); relato de dor (ausente, presente); relato de incômodo na boca (não, sim); satisfação com a vida (satisfeito, insatisfeito); satisfação com a saúde (satisfeito, insatisfeito).

O grupo das variáveis referente a hábitos contemplou: tabagismo (ausente, presente); etilismo (ausente, presente); uso de fio dental (sim, não); higiene bucal ( 2 ou mais vezes ao dia, menos de 2 vezes ao dia); autoexame da boca (sim, não).

Inicialmente, medidas de validade como sensibilidade (s), especificidade (e), acurácia, valor preditivo positivo (VPP) e valor preditivo negativo (VPN) foram calculadas para a autopercepção da presença da cárie dentária, tendo como referência ("padrão-ouro") a presença da cárie dentária normativa, ou seja, aferida clinicamente por um cirurgião-dentista devidamente calibrado.

Para este estudo, foram excluídos das análises os desdentados ( $\mathrm{n}=13)$, os sem informação sobre a autopercepção da presença da cárie dentária $(\mathrm{n}=18)$ e/ou os sem informação quanto à presença de cárie normativa $(\mathrm{n}=15)$. Sequencialmente, conduziu-se análise descritiva de todas as variáveis. Foram apresentados números absolutos e percentuais corrigidos pelo efeito de desenho para as categorias das variáveis independentes. Foi realizada análise bivariada através do teste qui-quadrado, e todas as variáveis independentes que se mostraram associadas à dependente ao nível de $20 \%(\mathrm{p} \leq 0,2)$ foram selecionadas para compor modelos múltiplos. Para a construção do modelo múltiplo, adotou-se o procedimento passo a passo, de forma que o modelo foi sendo ajustado. As variáveis que não estavam significativamente associadas e não contribuíam para o modelo foram eliminadas uma a uma. No modelo final, foram mantidos apenas as variáveis que permaneceram associadas ao nível de significância de $5 \%(\mathrm{p} \leq 0,05)$. O percentual da variância explicada pelo modelo foi calculado pelo coeficiente de determinação ajustado (pseudo R2).

O mesmo procedimento foi novamente conduzido considerando apenas os indivíduos que, de fato, apresentaram lesões normativas de cárie (Modelo 2) e, separadamente, considerando somente aqueles que não apresentaram tais lesões (Modelo 3). Nesses casos, a variável "presença de cárie dentária” foi excluída das análises. 
Todas as análises foram corrigidas pelo efeito do desenho, utilizando o comando complex samples do programa estatístico PASW 17.0.

\section{Resultados}

Foram utilizados dados dos 795 adultos considerando os conglomerados sorteados. A taxa de resposta foi de $91 \%$. Quanto às medidas de validade relativas à autopercepção da presença de cárie dentária, encontrou-se sensibilidade de 77,7\%, especificidade de 58\%, acurácia de 65\%, VPP de 52\% e VPN de $81 \%$.

Houve um predomínio de indivíduos na faixa etária de 35 a 39 anos, do sexo feminino, que se autodeclararam não brancos, que usaram serviços odontológicos não públicos e há mais de um ano. Nas condições normativas de saúde bucal, identificou-se que a maioria não possui lesão/lesões de cárie dentária e não apresentou necessidade de tratamento imediato/urgência. No entanto, há um predomínio das pessoas que autopercebem a presença da cárie dentária e acreditam necessitar de tratamento odontológico. Aproximadamente dois terços relataram não ser tabagista, não ser etilista e realizar a higiene bucal duas ou mais vezes ao dia, contudo cerca de um quinto da amostra tem o hábito de realizar o autoexame da boca. As demais informações segundo características sociodemográficas, utilização de serviço, normativas, de autopercepção e hábitos estão na Tabela 1.

Os resultados da análise bivariada, apresentados na Tabela 2, mostram que a maioria das variáveis independentes foi estatisticamente associada à variável dependente. Apenas a idade, cor da pele autodeclarada, estado civil e satisfação com a vida não foram associados ao nível de $\mathrm{p} \leq 0,2$ e, portanto, não entraram na composição do modelo múltiplo.

A análise múltipla foi construída sobre três perspectivas, apresentadas nos modelos 1, 2 e 3 (Tabela 3). O Modelo 1 apresenta o modelo múltiplo relativo à autopercepção da presença da cárie dentária conduzida entre todos os indivíduos $(\mathrm{n}=795)$. Identificou-se que houve maior chance de autopercepção da presença da cárie dentária entre os indivíduos que utilizaram os serviços odontológicos há mais de um ano, entre os que não receberam informações sobre como evitar problemas bucais, entre os que apresentavam lesões normativas de cárie dentária, entre os que autoperceberam necessidade de tratamento odontológico, entre os que relataram insatisfação com a saúde bucal, entre os que relataram dor, entre os que estavam insatisfeitos com a saúde geral e entre os que não usavam fio dental. Esse modelo foi capaz de explicar 41,2\% da variabilidade da autopercepção da presença da cárie dentária (pseudo R2).

O Modelo 2 traz o modelo múltiplo relativo à autopercepção da presença da cárie dentária conduzido somente entre os indivíduos que de fato apresentavam lesão de cárie dentária $(\mathrm{n}=295)$. Nesse caso, a chance de autopercepção da cárie dentária foi maior entre os que usaram o serviço há mais de um ano, os que perceberam necessidade de tratamento odontológico e entre os que estavam insatisfeitos com a saúde bucal e geral. O modelo foi capaz de explicar 27,3\% da variabilidade da autopercepção da presença da cárie dentária (pseudo R2).

O Modelo 3 revela o modelo múltiplo relativo à autopercepção da presença da cárie conduzido somente entre os indivíduos que não possuíam lesões normativas de cárie ( $\mathrm{n}=500)$. Entre eles, a chance de autopercepção da presença da cárie dentária foi maior entre os que usaram os serviços odontológicos há mais de um ano, entre os que não receberam informações sobre como evitar os problemas bucais, os que perceberam a necessidade de tratamento odontológico, os que estavam insatisfeitos com a saúde bucal e entre os que não usavam o fio dental. Esse modelo foi capaz de explicar 35\% da variabilidade da autopercepção da presença da cárie dentária (pseudo R2).

\section{Discussão}

A validade de testes diagnósticos é um importante aspecto a ser considerado quando se enfoca a eficácia das medidas de autopercepção em prever desfechos normativos de saúde. Medidas de autopercepção sofrem influência de variáveis contextuais e individuais, variando de indivíduo para indivíduo ou no mesmo indivíduo em diferentes momentos. Apesar da subjetividade inerente a tais 


\section{Tabela 1}

Distribuição dos adultos em números absolutos (n) e percentuais corrigidos pelo efeito de desenho (\%) segundo características sociodemográficas, utilização de serviço, normativas, de autopercepção e hábitos. Montes Claros, Minas Gerais, Brasil, 2009 ( $n=795$ ).

\begin{tabular}{|c|c|c|}
\hline Variáveis & $\mathbf{n}$ & $\%$ \\
\hline \multicolumn{3}{|l|}{ Sociodemográficas } \\
\hline \multicolumn{3}{|l|}{ Idade (anos) } \\
\hline $35-39$ & 424 & 53,3 \\
\hline $40-44$ & 371 & 46,7 \\
\hline \multicolumn{3}{|l|}{ Sexo } \\
\hline Feminino & 427 & 53,7 \\
\hline Masculino & 368 & 46,3 \\
\hline \multicolumn{3}{|l|}{ Cor da pele autodeclarada } \\
\hline Branco & 222 & 27,9 \\
\hline Não branco & 573 & 72,1 \\
\hline \multicolumn{3}{|l|}{ Local de residência } \\
\hline Zona urbana & 780 & 98,1 \\
\hline Zona rural & 15 & 1,9 \\
\hline \multicolumn{3}{|l|}{ Renda per capita ${ }^{*, * *}$} \\
\hline$>\mathrm{R} \$ 200,00$ & 404 & 52,7 \\
\hline$\leq \mathrm{R} \$ 200,00$ & 363 & 47,3 \\
\hline \multicolumn{3}{|l|}{ Escolaridade (anos) } \\
\hline$>8$ & 454 & 57,1 \\
\hline$\leq 8$ & 331 & 41,6 \\
\hline Analfabeto/Sem escolaridade & 10 & 1,3 \\
\hline \multicolumn{3}{|l|}{ União estável } \\
\hline Sim & 586 & 73,7 \\
\hline Não & 209 & 26,3 \\
\hline \multicolumn{3}{|l|}{ Posse de automóvel } \\
\hline Possui & 256 & 32,2 \\
\hline Não possui & 539 & 67,8 \\
\hline \multicolumn{3}{|l|}{ Utilização de serviços odontológicos } \\
\hline \multicolumn{3}{|l|}{ Tipo de serviço *** } \\
\hline Não público & 516 & 65,6 \\
\hline Público & 270 & 34,4 \\
\hline \multicolumn{3}{|c|}{ Tempo do último uso do serviço odontológicos (anos) ${ }^{* \star \star}$} \\
\hline$<1$ & 369 & 46,9 \\
\hline$>1$ & 417 & 53,1 \\
\hline \multicolumn{3}{|l|}{ Motivo do uso *** } \\
\hline Rotina/Manutenção & 280 & 35,6 \\
\hline Problemas odontológicos & 506 & 64,4 \\
\hline \multicolumn{3}{|c|}{ Recebeu informações sobre como evitar problemas bucais * } \\
\hline Sim & 501 & 63,6 \\
\hline Não & 287 & 36,4 \\
\hline
\end{tabular}

(continua) 
Tabela 1 (continuação)

\begin{tabular}{|c|c|c|}
\hline Variáveis & $\mathbf{n}$ & $\%$ \\
\hline \multicolumn{3}{|c|}{ Condições normativas } \\
\hline \multicolumn{3}{|c|}{ Número de dentes presentes } \\
\hline$\geq 20$ & 629 & 79,1 \\
\hline$<20$ & 166 & 20,9 \\
\hline \multicolumn{3}{|c|}{ Cárie dentária normativa } \\
\hline Ausente & 500 & 62,9 \\
\hline Presente & 295 & 37,1 \\
\hline \multicolumn{3}{|c|}{ Doença periodontal } \\
\hline Ausente & 719 & 90,4 \\
\hline Presente & 76 & 9,6 \\
\hline \multicolumn{3}{|c|}{ Lesão na mucosa * } \\
\hline Ausente & 689 & 86,9 \\
\hline Presente & 104 & 13,1 \\
\hline \multicolumn{3}{|c|}{ Necessidade de tratamento de urgência } \\
\hline Não & 663 & 83,4 \\
\hline $\operatorname{sim}$ & 132 & 16,6 \\
\hline
\end{tabular}

(continua)

medidas, ainda assim, elas têm sido usadas e recomendadas pela literatura científica 1,3,5,8,10. Logo, é necessário quantificar até que ponto reproduzem estados normativos, ou até que ponto os itens de autorrelato medem o que pretendem medir ${ }^{17}$. Neste estudo foi observado que, do total dos pacientes com a presença normativa de lesões de cárie, 78\% autoperceberam essa condição (sensibilidade). Os poucos estudos prévios identificados que abordaram essa questão evidenciaram sensibilidade para a autopercepção da cárie tanto mais alta (84\%) 11, quanto mais baixa (59\%) 22. Já do total de indivíduos que não apresentou lesões normativas de cárie dentária, apenas 58\% se perceberam livres de lesões de cárie (especificidade), revelando que muitos que acreditam possuir a lesão, na realidade não a apresentam. Estudos prévios encontraram especificidades de 100\% 11 entre usuários da clínica odontológica da Faculdade de Odontologia da Universidade Estadual Paulista (Araraquara, São Paulo) e de 85,7\% entre pacientes da Harvard School of Dental Medicine (Boston, Estados Unidos) 22. O fato de a amostra investigada neste estudo não ser proveniente de usuários de um serviço odontológico, mas sim adultos da comunidade em geral, pode ser a explicação para essas divergências. Dados obtidos de pessoas que frequentam um serviço de saúde possivelmente sejam diferentes de dados levantados em situações do cotidiano comunitário, pois quem está em um serviço de saúde tende a perceber mais suas condições de morbidade ou de necessidade de tratamento, já que o ambiente clínico representa importante oportunidade para aprendizagem e de maior conscientização de sua condição bucal 23 . Apesar dessa diferença quanto à população investigada, esses foram os únicos estudos localizados 11,22 que buscaram validar medidas de autopercepção da cárie dentária. Portanto, essas comparações precisam ser feitas com cautela.

Diferenças verificadas nos resultados de diferentes investigações podem também ser atribuídas a questões culturais e de escolaridade, fatores que sabidamente influenciam a autopercepção 1,8, mas que limitam a utilização em ampla escala dos autorrelatos da presença de lesões de cárie enquanto teste diagnóstico, o que não significa dizer que não apresente utilidade.

Em contextos específicos, como em populações emancipadas; grupos fechados bem esclarecidos, que estejam recebendo informações/instruções constantes em saúde bucal e sendo estimuladas ao autoexame bucal, as medidas de autopercepção poderiam revelar melhores níveis de validade, tal como foi verificado entre usuários de clínica odontológica no contexto universitário 11.

O diagnóstico da cárie dentária é complexo, mesmo ao se adotarem outros métodos menos subjetivos. Um estudo, realizado para comparar métodos de diagnóstico clínico de cárie oclusal in vitro 24, revelou uma sensibilidade de $67 \%$ para a inspeção visual e de $58 \%$ para a inspeção visual associada 
Tabela 1 (continuação)

\begin{tabular}{|c|c|c|}
\hline Variáveis & $\mathbf{n}$ & $\%$ \\
\hline \multicolumn{3}{|l|}{ Autopercepção } \\
\hline \multicolumn{3}{|c|}{ Autopercepção de presença de cárie dentária } \\
\hline Não & 356 & 44,8 \\
\hline Sim & 439 & 55,2 \\
\hline \multicolumn{3}{|c|}{ Autopercepção da necessidade de tratamento odontológico * } \\
\hline Não & 158 & 19,9 \\
\hline Sim & 635 & 80,1 \\
\hline \multicolumn{3}{|l|}{ Autopercepção da aparência } \\
\hline Excelente/Boa & 429 & 54,0 \\
\hline Regular/Ruim/Péssima & 366 & 46,0 \\
\hline \multicolumn{3}{|c|}{ Autopercepção da mastigação } \\
\hline Excelente/Boa & 466 & 58,6 \\
\hline Regular/Ruim/Péssima & 329 & 41,4 \\
\hline \multicolumn{3}{|l|}{ Autopercepção da fala } \\
\hline Excelente/Boa & 654 & 82,3 \\
\hline Regular/Ruim/Péssima & 141 & 17,7 \\
\hline \multicolumn{3}{|c|}{ Autopercepção do relacionamento social } \\
\hline Não afeta & 549 & 69,1 \\
\hline Afeta & 246 & 30,9 \\
\hline \multicolumn{3}{|l|}{ Autopercepção do paladar } \\
\hline Excelente/Boa & 735 & 92,5 \\
\hline Regular/Ruim/Péssima & 60 & 7,5 \\
\hline \multicolumn{3}{|c|}{ Autopercepção de mau hálito } \\
\hline Não Percebido & 712 & 89,6 \\
\hline Percebido & 83 & 10,4 \\
\hline \multicolumn{3}{|c|}{ Autopercepção da saúde bucal } \\
\hline Satisfeito & 392 & 49,3 \\
\hline Insatisfeito & 403 & 50,7 \\
\hline \multicolumn{3}{|l|}{ Relato de dor } \\
\hline Ausente & 486 & 61,1 \\
\hline Presente & 309 & 38,9 \\
\hline \multicolumn{3}{|l|}{ Relato de incômodo na boca } \\
\hline Não & 635 & 79,9 \\
\hline Sim & 160 & 20,1 \\
\hline \multicolumn{3}{|l|}{ Satisfação com a vida } \\
\hline Satisfeito & 685 & 86,2 \\
\hline Insatisfeito & 110 & 13,8 \\
\hline \multicolumn{3}{|l|}{ Satisfação com a saúde geral } \\
\hline Satisfeito & 275 & 34,6 \\
\hline Insatisfeito & 520 & 65,4 \\
\hline
\end{tabular}

(continua)

à radiografia interproximal, considerando o exame histológico como "padrão-ouro". Em relação à especificidade, os métodos mais específicos foram a associação da inspeção visual com a radiografia interproximal (90\%) e a transiluminação por fibra óptica (90\%). A radiografia digital direta mostrou baixa especificidade (30\%) 24. Tais achados evidenciam que outros testes diagnósticos de cárie dentária também apresentam valores relativamente modestos de validade. Entretanto, novamente, a comparação de tais resultados com os de autopercepção deve ser avaliada com cautela, já que os exames utilizados como "padrão-ouro" são bem diferentes entre os estudos. 
Tabela 1 (continuação)

\begin{tabular}{lll}
\hline Variáveis & $\mathbf{n}$ & $\%$ \\
\hline Hábitos & & \\
$\quad$ Tabagismo & 605 & 76,1 \\
$\quad$ Ausente & 190 & 23,9 \\
$\quad$ Presente & & 57,4 \\
Etilismo & 456 & 42,6 \\
$\quad$ Ausente & 339 & \\
$\quad$ Presente & & 53,2 \\
Uso de fio dental & 423 & 46,8 \\
$\quad$ Usa & 372 & 63,5 \\
$\quad$ Não usa & & 36,5 \\
Higiene bucal (vezes ao dia) & 505 & \\
$\quad \geq 2$ & 290 & 20,4 \\
$\quad<2$ & & 79,6 \\
Autoexame da boca * & 162 & \\
$\quad$ Sim & 632 & \\
Não & & \\
\hline
\end{tabular}

* Variação no $n$ de 795 devido à perda de informação;

** Cotação do dólar à época da coleta de dados: US $\$ 1,00=\mathrm{R} \$ 2,07$;

*** Foram excluídos os 9 indivíduos $(1,4 \%)$ que nunca usaram os serviços odontológicos.

A sensibilidade e a especificidade são calculadas com base em um teste de referência ("padrão-ouro"), contudo, na prática, quando um teste é utilizado com finalidade diagnóstica, não se sabe previamente quem são os indivíduos portadores ou não de determinado agravo 25 . Por conseguinte, na prática clínica e para fins epidemiológicos, é importante observar mais o valor preditivo do teste do que sua sensibilidade e especificidade, visto que o valor preditivo indica a proporção de indivíduos que de fato apresentam ou não a lesão diante de um resultado do teste 25 .

O VPP foi relativamente baixo (52\%) e indica que, dentre os indivíduos que autoperceberam presença de cárie dentária, apenas a metade realmente apresentava tais lesões. Possivelmente, outros problemas bucais podem estar sendo equivocadamente percebidos como cárie dentária, evidenciando superestimação na percepção da presença da cárie dentária. A literatura científica já confirmou maior fidedignidade nos relatos de autopercepção da cárie do que nos relatos de autopercepção da doença periodontal 10,22. Estudo prévio 1 encontrou maiores discrepâncias entre índices clínicos e percepção das condições bucais entre idosos, sendo a diferença maior na avaliação das doenças periodontais do que na cárie dentária. Investigação conduzida entre universitários do Japão 26 revelou que a má oclusão e sintomas de disfunção temporomandibular (DTM) apresentaram pouca influência sobre a autopercepção da saúde bucal, enquanto a experiência de cárie dentária afetou significativamente essa autopercepção. A precária percepção de outros problemas bucais pode favorecer a percepção de presença de cárie, por ser o problema bucal mais prevalente e por existir maior conhecimento popular a respeito das lesões cariosas do que qualquer outro tipo de alteração presente na cavidade bucal.

O presente estudo considerou algumas outras condições normativas nas análises (doença periodontal, número de dentes presentes, lesões de mucosa e atendimento de urgência). Entretanto, nenhuma dessas condições avaliadas manteve-se associada à autopercepção da presença de cárie dentária nos modelos múltiplos ajustados. Estudos futuros poderiam melhor elucidar essa relação, ao contemplarem manchamentos/pigmentações dos dentes, fraturas, halitose, má oclusão, entre outras potenciais condições que possam estar sendo percebidas como cárie dentária.

Por outro lado, foi constatado VPN relativamente alto (81\%). Assim, dentre os que afirmaram não autoperceberem cárie dentária, $81 \%$ de fato não possuíam a doença, o que torna a percepção de ausência da cárie dentária mais confiável. Novamente, por ser o problema bucal mais prevalente, por existir 
Tabela 2

Distribuição dos adultos em números absolutos (n) e percentuais corrigidos pelo efeito de desenho (\%) segundo análise bivariada da autopercepção da cárie dentária e sua associação com características sociodemográficas, utilização de serviço, normativas, de autopercepção e hábitos. Montes Claros, Minas Gerais, Brasil, 2009 ( $n=795$ ).

\begin{tabular}{|c|c|c|c|c|c|}
\hline \multirow[t]{3}{*}{ Variáveis } & \multicolumn{4}{|c|}{ Autopercepção da cárie dentária } & \multirow[t]{3}{*}{ Valor de $p$} \\
\hline & \multirow[b]{2}{*}{$\mathbf{n}$} & Não & \multicolumn{2}{|c|}{ Sim } & \\
\hline & & $\%$ & $\mathbf{n}$ & $\%$ & \\
\hline \multicolumn{6}{|l|}{ Sociodemográficas } \\
\hline \multicolumn{6}{|l|}{ Idade (anos) } \\
\hline $35-39$ & 186 & 43,9 & 238 & 56,1 & 0,58 \\
\hline $40-44$ & 170 & 45,8 & 201 & 54,2 & \\
\hline \multicolumn{6}{|l|}{ Sexo } \\
\hline Feminino & 208 & 48,7 & 219 & 51,3 & 0,016 \\
\hline Masculino & 148 & 40,2 & 220 & 59,8 & \\
\hline \multicolumn{6}{|l|}{ Cor da pele autodeclarada } \\
\hline Branco & 107 & 48,2 & 115 & 51,8 & 0,228 \\
\hline Não branco & 249 & 43,5 & 324 & 56,5 & \\
\hline \multicolumn{6}{|l|}{ Local de residência } \\
\hline Zona urbana & 353 & 45,3 & 427 & 54,7 & 0,051 \\
\hline Zona rural & 3 & 20,0 & 12 & 80,0 & \\
\hline \multicolumn{6}{|l|}{ Renda per capita *,** } \\
\hline$>\mathrm{R} \$ 200,00$ & 224 & 55,4 & 180 & 44,6 & $<0,001$ \\
\hline$\leq \mathrm{R} \$ 200,00$ & 123 & 33,9 & 240 & 66,1 & \\
\hline \multicolumn{6}{|l|}{ Escolaridade (anos) } \\
\hline$>8$ & 248 & 54,6 & 206 & 45,4 & $<0,001$ \\
\hline$\leq 8$ & 105 & 31,7 & 226 & 68,3 & \\
\hline Analfabeto/Sem escolaridade & 3 & 30,0 & 7 & 70,0 & \\
\hline \multicolumn{6}{|l|}{ União estável } \\
\hline Sim & 268 & 45,7 & 318 & 54,3 & 0,365 \\
\hline Não & 88 & 42,1 & 121 & 57,9 & \\
\hline \multicolumn{6}{|l|}{ Posse de automóvel } \\
\hline Possui automóvel & 143 & 55,9 & 113 & 44,1 & $<0,001$ \\
\hline Não possui automóvel & 213 & 39,5 & 326 & 60,5 & \\
\hline \multicolumn{6}{|l|}{ Utilização de serviços odontológicos } \\
\hline \multicolumn{6}{|l|}{ Tipo de serviço *** } \\
\hline Não público & 248 & 48,1 & 268 & 51,9 & 0,024 \\
\hline Público & 107 & 39,6 & 163 & 60,4 & \\
\hline \multicolumn{6}{|c|}{ 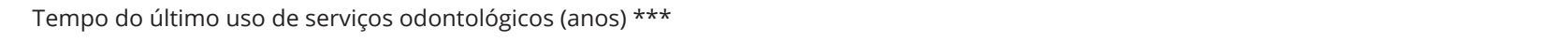 } \\
\hline$<1$ & 214 & 58,0 & 155 & 42,0 & $<0,001$ \\
\hline$>1$ & 141 & 33,8 & 276 & 66,2 & \\
\hline \multicolumn{6}{|l|}{ Motivo do uso *** } \\
\hline Rotina/Manutenção & 164 & 58,6 & 116 & 41,4 & $<0,001$ \\
\hline Problemas odontológicos & 191 & 37,7 & 315 & 62,3 & \\
\hline \multicolumn{6}{|c|}{ Recebeu informações sobre como evitar problemas bucais * } \\
\hline Sim & 267 & 53,3 & 234 & 46,7 & $<0,001$ \\
\hline Não & 87 & 30,3 & 200 & 69,7 & \\
\hline
\end{tabular}

(continua) 
Tabela 2 (continuação)

\begin{tabular}{|c|c|c|c|c|c|}
\hline \multirow[t]{3}{*}{ Variáveis } & \multicolumn{4}{|c|}{ Autopercepção da cárie dentária } & \multirow[t]{3}{*}{ Valor de $p$} \\
\hline & \multicolumn{2}{|c|}{ Não } & \multicolumn{2}{|c|}{ Sim } & \\
\hline & $\mathbf{n}$ & $\%$ & $\mathbf{n}$ & $\%$ & \\
\hline \multicolumn{6}{|c|}{ Condições normativas } \\
\hline \multicolumn{6}{|c|}{ Número de dentes presentes } \\
\hline$\geq 20$ & 292 & 46,4 & 337 & 53,6 & 0,07 \\
\hline$<20$ & 64 & 38,6 & 102 & 61,4 & \\
\hline \multicolumn{6}{|c|}{ Cárie dentária normativa } \\
\hline Ausente & 290 & 58,0 & 210 & 42,0 & $<0,001$ \\
\hline Presente & 66 & 22,4 & 229 & 77,6 & \\
\hline \multicolumn{6}{|c|}{ Doença periodontal } \\
\hline Ausente & 338 & 47,0 & 381 & 53,0 & $<0,001$ \\
\hline Presente & 18 & 23,7 & 58 & 76,3 & \\
\hline \multicolumn{6}{|c|}{ Lesão na mucosa * } \\
\hline Ausente & 318 & 46,2 & 371 & 53,8 & 0,027 \\
\hline Presente & 36 & 34,6 & 68 & 65,4 & \\
\hline \multicolumn{6}{|c|}{ Necessidade de tratamento de urgência } \\
\hline Não & 323 & 48,7 & 340 & 51,3 & $<0,001$ \\
\hline Sim & 33 & 25,0 & 99 & 75,0 & \\
\hline
\end{tabular}

(continua)

maior conhecimento popular a seu respeito e, sobretudo por ser a alteração dentária mais perceptível visualmente, os relatos de sua ausência sejam bem mais fidedignos do que de sua presença. Perseguir VPN ainda mais altos, pela adoção de medidas que garantam maior e melhor acesso à informação em saúde bucal à população, poderia, no futuro, colocar a autopercepção como uma potencial ferramenta de triagem 10 para a necessidade de tratamento para a cárie dentária, em que somente os que percebessem lesões necessitariam de exames clínicos confirmatórios.

A autopercepção da presença de cárie dentária foi maior entre os que utilizaram os serviços odontológicos há mais tempo, autoperceberam necessidade de tratamento odontológico e relataram insatisfação com a saúde bucal, independentemente de apresentarem ou não lesões normativas (em todos os modelos). No geral, constatou-se que a percepção da presença de cárie esteve associada a contextos desfavoráveis, embora não tenha revelado associação com nenhuma variável sociodemográfica.

O tempo de uso dos serviços odontológicos superior a um ano esteve associado a uma maior autopercepção em todos os modelos. O uso dos serviços odontológicos há mais tempo pode piorar as condições normativas de saúde bucal, bem como aumentar a insegurança dos indivíduos com sua própria saúde. Também já foi evidenciado que o uso dos serviços odontológicos no último ano esteve associado a percepções mais positivas da saúde bucal entre adultos 27 e idosos 28 . Assim sendo, o uso dos serviços odontológicos com frequência adequada poderia aumentar o poder preditivo da autopercepção da saúde bucal e merece ser perseguido.

A variável que mais fortemente esteve associada à autopercepção da presença da cárie dentária nos três modelos avaliados foi autopercepção da necessidade de tratamento odontológico, corroborando com a ideia de que muitos dos problemas bucais podem estar sendo atribuídos, ainda que de forma equivocada, à cárie dentária. Da mesma forma, a autopercepção negativa da saúde bucal também mostrou associação com o desfecho em todos os modelos. Já foi constatado que o principal motivo relatado para a procura do serviço odontológico foi a presença de lesões normativas de cárie 29. Estudos prévios também já evidenciaram que a autopercepção negativa das condições de saúde bucal representa o fator preditor essencial da procura por atendimento odontológico 1,5,8, sendo destacada como um fator determinante de saúde bucal em países com os sistemas de saúde ainda em desenvolvimento 30. Embora variáveis subjetivas como as de autopercepção abriguem certas limitações 1,3, os serviços de saúde não podem desprezar tal conjunto de informações, pois apresentam o potencial de 
Tabela 2 (continuação)

\begin{tabular}{|c|c|c|c|c|c|}
\hline \multirow[t]{3}{*}{ Variáveis } & \multicolumn{4}{|c|}{ Autopercepção da cárie dentária } & \multirow[t]{3}{*}{ Valor de $\mathrm{p}$} \\
\hline & \multirow[b]{2}{*}{$\mathbf{n}$} & Não & \multicolumn{2}{|c|}{ Sim } & \\
\hline & & $\%$ & $\mathbf{n}$ & $\%$ & \\
\hline \multicolumn{6}{|l|}{ Autopercepção } \\
\hline \multicolumn{6}{|c|}{ Autopercepção da necessidade de tratamento odontológico * } \\
\hline Não & 132 & 83,5 & 26 & 16,5 & $<0,001$ \\
\hline Sim & 222 & 35,0 & 413 & 65,0 & \\
\hline \multicolumn{6}{|l|}{ Autopercepção da aparência } \\
\hline Excelente/Boa & 239 & 55,7 & 190 & 44,3 & $<0,001$ \\
\hline Regular/Ruim/Péssima & 117 & 32,0 & 249 & 68,0 & \\
\hline \multicolumn{6}{|c|}{ Autopercepção da mastigação } \\
\hline Excelente/Boa & 246 & 52,8 & 220 & 47,2 & $<0,001$ \\
\hline Regular/Ruim/Péssima & 110 & 33,4 & 219 & 66,6 & \\
\hline \multicolumn{6}{|l|}{ Autopercepção da fala } \\
\hline Excelente/Boa & 313 & 47,9 & 341 & 52,1 & $<0,001$ \\
\hline Regular/Ruim/Péssima & 43 & 30,5 & 98 & 69,5 & \\
\hline \multicolumn{6}{|c|}{ Autopercepção do relacionamento social } \\
\hline Não afeta & 269 & 49,0 & 280 & 51,0 & $<0,001$ \\
\hline Afeta & 87 & 35,4 & 159 & 64,6 & \\
\hline \multicolumn{6}{|l|}{ Autopercepção do paladar } \\
\hline Excelente/Boa & 342 & 46,5 & 393 & 53,5 & 0,001 \\
\hline Regular/Ruim/Péssima & 14 & 23,3 & 46 & 76,7 & \\
\hline \multicolumn{6}{|c|}{ Autopercepção de mau hálito } \\
\hline Não percebido & 335 & 47,1 & 377 & 52,9 & $<0,001$ \\
\hline Percebido & 21 & 25,3 & 62 & 74,7 & \\
\hline \multicolumn{6}{|c|}{ Autopercepção da saúde bucal } \\
\hline Satisfeito & 246 & 62,8 & 146 & 37,2 & $<0,001$ \\
\hline Insatisfeito & 110 & 27,3 & 293 & 72,7 & \\
\hline \multicolumn{6}{|l|}{ Relato de dor } \\
\hline Ausente & 261 & 53,7 & 225 & 46,3 & $<0,001$ \\
\hline Presente & 95 & 30,7 & 214 & 69,3 & \\
\hline \multicolumn{6}{|l|}{ Relato de incômodo na boca } \\
\hline Não & 301 & 47,4 & 334 & 52,6 & 0,003 \\
\hline Sim & 55 & 34,4 & 105 & 65,6 & \\
\hline \multicolumn{6}{|l|}{ Satisfação com a vida } \\
\hline Satisfeito & 310 & 45,3 & 375 & 54,7 & 0,501 \\
\hline Insatisfeito & 46 & 41,8 & 64 & 58,2 & \\
\hline \multicolumn{6}{|l|}{ Satisfação com a saúde geral } \\
\hline Satisfeito & 168 & 61,1 & 107 & 38,9 & $<0,001$ \\
\hline Insatisfeito & 188 & 36,2 & 332 & 63,8 & \\
\hline
\end{tabular}

(continua)

identificar grupos que percebem de modo negativo suas condições de saúde e que podem, efetivamente, apresentar pior estado de saúde 18. Dessa maneira, acessar tais percepções contribuiria com maior equidade por parte dos serviços de saúde.

A autopercepção da presença de cárie dentária mostrou-se associada à pior percepção de saúde geral somente nos modelos 1 e 2 , sugerindo que, mais do que a percepção em si, a presença de lesões normativas impactaria na satisfação da saúde geral porque não se mostrou associada entre aqueles que percebiam tais lesões, sem realmente as possuírem (Modelo 3). Estudo prévio verificou que a qualidade de vida e a percepção da saúde geral sofrem influência de condições bucais precárias 31 e 
Tabela 2 (continuação)

\begin{tabular}{|c|c|c|c|c|c|}
\hline \multirow[t]{3}{*}{ Variáveis } & \multicolumn{4}{|c|}{ Autopercepção da cárie dentária } & \multirow[t]{3}{*}{ Valor de $p$} \\
\hline & \multicolumn{2}{|c|}{ Não } & \multicolumn{2}{|c|}{ Sim } & \\
\hline & $\mathbf{n}$ & $\%$ & $\mathbf{n}$ & $\%$ & \\
\hline \multicolumn{6}{|l|}{ Hábitos } \\
\hline \multicolumn{6}{|l|}{ Tabagismo } \\
\hline Ausente & 293 & 48,4 & 312 & 51,6 & $<0,001$ \\
\hline Presente & 63 & 33,2 & 127 & 66,8 & \\
\hline \multicolumn{6}{|l|}{ Etilismo } \\
\hline Ausente & 223 & 48,9 & 233 & 51,1 & 0,007 \\
\hline Presente & 133 & 39,2 & 206 & 60,8 & \\
\hline \multicolumn{6}{|c|}{ Uso de fio dental } \\
\hline Usa & 239 & 56,5 & 184 & 43,5 & $<0,001$ \\
\hline Não usa & 117 & 31,5 & 255 & 68,5 & \\
\hline \multicolumn{6}{|c|}{ Higiene bucal (vezes ao dia) } \\
\hline$\geq 2$ & 268 & 53,1 & 237 & 46,9 & $<0,001$ \\
\hline$<2$ & 88 & 30,3 & 202 & 69,7 & \\
\hline \multicolumn{6}{|c|}{ Autoexame da boca * } \\
\hline Sim & 81 & 50,0 & 81 & 50,0 & 0,139 \\
\hline Não & 275 & 43,5 & 357 & 56,5 & \\
\hline
\end{tabular}

* Variação no n de 795 devido à perda de informação;

** Cotação do dólar à época da coleta de dados: US\$1,00 = R \$2,07;

*** Foram excluídos os 9 indivíduos $(1,4 \%)$ que nunca usaram os serviços odontológicos.

Nota: foram destacadas (negrito) as variáveis selecionadas para compor o modelo múltiplo $(p<0,2)$.

da pior autopercepção da saúde bucal de adultos 32 e idosos 33. Por outro lado, a insatisfação com a própria saúde poderia exacerbar a autopercepção negativa da saúde bucal, uma vez que a depressão já foi considerada como um preditor de autoavaliação negativa da saúde bucal por estudo prévio 34 . Como se trata de um estudo transversal, não se pode afirmar a direção das associações observadas, porém, fica evidenciado que saúde bucal e geral não são percebidas de forma isolada, e a percepção negativa de um desses fatores provavelmente interfere no outro.

Estudo prévio 11 constatou efetividade da autopercepção da cárie dentária em predizer a presença normativa de lesões em pacientes atendidos por um serviço odontológico universitário. A chance de autopercepção da presença da cárie dentária foi três vezes maior entre os indivíduos que de fato apresentavam lesões, quando comparados aos que não apresentavam (Modelo 1). Esse achado enfatiza a importância das questões relativas à autopercepção da saúde, pois os autorrelatos dos pacientes apresentam o potencial de se tornar instrumentos de rastreamento, de uma modo que mais indivíduos considerem aceitável e com menor demanda de tempo e custo 10,11, sendo também recomendável sua consideração na avaliação dos indivíduos para atendimento odontológico ${ }^{11}$. Além disso, outros estudos já demonstraram a eficácia de se mensurar os níveis de saúde bucal por intermédio de escalas de autorrelato 3,35 , no entanto são escassos os estudos voltados especificamente para a validade da autopercepção da cárie dentária, tendo sido identificados apenas dois estudos nessa temática 11,22.

Os adultos investigados que relataram dor, independentemente de possuírem ou não lesões normativas (Modelo 1), apresentaram uma chance maior de autopercepção da presença de cárie dentária. A dor é uma reconhecida medida de impacto da saúde bucal na qualidade de vida, e estudos prévios já confirmaram a dor como preditora da autopercepção negativa da saúde bucal 28,35. Na ausência desse sintoma, muitos acreditam estar livres de doenças bucais 4 e, sendo a cárie dentária a mais prevalente doença bucal, a presença de dor pode ser interpretada como presença de cárie dentária. Esse achado sugere que sintomas subjetivamente percebidos podem acabar sendo interpretados pelos indivíduos como indicadores de doença, independentemente da presença de sinais clínicos, objetivamente conferidos. 


\section{Tabela 3}

Modelos múltiplos de regressão logística binária ajustados relativos à autopercepção da presença da cárie dentária entre adultos.

\begin{tabular}{|c|c|c|c|c|c|c|}
\hline \multirow[t]{2}{*}{ Variáveis } & \multicolumn{2}{|c|}{ Modelo 1 * } & \multicolumn{2}{|c|}{ Modelo 2 ** } & \multicolumn{2}{|c|}{ Modelo $3 * * *$} \\
\hline & OR (IC95\%) & Valor de $p$ & OR (IC95\%) & Valor de $p$ & OR (IC95\%) & Valor de $p$ \\
\hline \multicolumn{7}{|c|}{ Tempo do último uso do serviço (anos) } \\
\hline$<1$ & 1,00 & & 1,00 & & 1,00 & \\
\hline$>1$ & $1,96(1,38 ; 2,78)$ & $<0,001$ & $2,33(1,29 ; 4,19)$ & 0,005 & $1,90(1,23 ; 2,93)$ & 0,005 \\
\hline \multicolumn{7}{|c|}{$\begin{array}{l}\text { Recebeu informações sobre como evitar } \\
\text { problemas bucais }\end{array}$} \\
\hline Sim & 1,00 & & - & - & 1,00 & \\
\hline Não & $1,84(1,27 ; 2,67)$ & 0,001 & - & - & $2,21(1,50 ; 3,25)$ & $<0,001$ \\
\hline \multicolumn{7}{|c|}{ Cárie dentária normativa } \\
\hline Ausente & 1,00 & & - & - & - & - \\
\hline Presente & $3,03(2,08 ; 4,42)$ & $<0,001$ & - & - & - & - \\
\hline \multicolumn{7}{|c|}{$\begin{array}{l}\text { Autopercepção da necessidade de } \\
\text { tratamento odontológico }\end{array}$} \\
\hline Não & 1,00 & & 1,00 & & 1,00 & \\
\hline Sim & $4,23(2,55 ; 6,99)$ & $<0,001$ & $5,03(1,73 ; 14,7)$ & 0,003 & $4,52(2,17 ; 9,41)$ & $<0,001$ \\
\hline \multicolumn{7}{|c|}{ Autopercepção da saúde bucal } \\
\hline Satisfeito & 1,00 & & 1,00 & & 1,00 & \\
\hline Insatisfeito & $2,18(1,52 ; 3,13)$ & $<0,001$ & $3,29(1,77 ; 6,12)$ & $<0,001$ & $2,81(1,64 ; 4,81)$ & $<0,001$ \\
\hline \multicolumn{7}{|l|}{ Relato de dor } \\
\hline Ausente & 1,00 & & - & - & - & - \\
\hline Presente & $1,51(1,04 ; 2,19)$ & 0,030 & - & - & - & - \\
\hline \multicolumn{7}{|c|}{ Satisfação com a saúde geral } \\
\hline Satisfeito & 1,00 & & 1,00 & & - & - \\
\hline Insatisfeito & $1,94(1,34 ; 2,80)$ & $<0,001$ & $2,88(1,54 ; 5,40)$ & 0,001 & - & - \\
\hline \multicolumn{7}{|c|}{ Uso de fio dental } \\
\hline Usa & 1,00 & & - & - & 1,00 & \\
\hline Não usa & $1,79(1,26 ; 2,55)$ & 0,001 & - & - & $2,94(1,69 ; 5,10)$ & $<0,001$ \\
\hline Intercepto $(\beta)$ & \multicolumn{2}{|l|}{$-3,154$} & \multicolumn{2}{|l|}{$-2,019$} & \multicolumn{2}{|l|}{2,205} \\
\hline Pseudo R2 & \multicolumn{2}{|l|}{$41,2 \%$} & \multicolumn{2}{|l|}{$27,3 \%$} & \multicolumn{2}{|l|}{$35 \%$} \\
\hline
\end{tabular}

* Modelo 1: foram incluídos todos os adultos ( $n=795)$;

** Modelo 2: foram incluídos apenas os adultos sabidamente portadores de lesões normativas de cárie dentária ( $\mathrm{n}=295)$;

*** Modelo 3: foram incluídos apenas os adultos que sabidamente não apresentavam lesões normativas de cárie dentária ( $\mathrm{n}=500$ ).

Nos modelos 1 e 3, adultos que não receberam informações sobre como evitar problemas bucais apresentaram chance aproximadamente duplicada de autoperceberem presença de cárie dentária. Foi interessante observar que tal associação ocorreu na ausência de lesões normativas (Modelo 3), indicando que adultos que não relataram acesso a informações perceberam de forma mais incoerente sua condição bucal (autoperceberam lesões de cárie, mesmo não as possuindo). Esse relevante achado evidencia a importância de garantir acesso a informações, em quantidade e qualidade adequadas, para estimular hábitos de autoexame e autocuidado, contribuindo para tornar os indivíduos mais conscientes e confiantes de sua condição bucal. Estudos prévios já verificaram que o acesso a informações contribui para melhores condições bucais normativas 36,37 e percebidas 38. Investigação recente constatou que baixos níveis de alfabetização em saúde, mesmo após ajuste por determinantes socioeconômicos e demográficos, foram preditores de uma pior autopercepção da saúde bucal 30 . Consequentemente, a garantia de maior acesso a informações em saúde bucal aponta como o principal caminho a ser perseguido para aumentar o poder preditivo da autopercepção das doenças bucais, dentre elas, a cárie dentária. 
Os adultos que relataram não utilizar o fio dental na higiene bucal referiram maior autopercepção da presença de cárie dentária (modelos 1 e 3). Estudo prévio demonstrou que piores hábitos de higiene bucal estiveram associados a uma pior percepção da saúde bucal 26 . Sabe-se que o uso do fio dental é um comportamento amplamente recomendado para se alcançar níveis satisfatórios de saúde bucal. A ausência dessa prática contribui, talvez, com maior autopercepção de problemas bucais porque comportamentos "inadequados" em saúde são consequentemente associados ao surgimento de lesões. Os resultados da presente investigação reforçam essa ideia, já que a ausência do uso do fio dental se mostrou associada à autopercepção da cárie dentária mesmo naqueles indivíduos que não apresentavam lesões normativas.

Convém destacar que o relato de uso do fio dental parece mais sensível para mensurar o autocuidado em saúde bucal do que o relato de frequência de escovação dentária. Em estudos conduzidos entre adultos 39,40, o relato de escovação dentária de três vezes ao dia é recorrente, podendo, em várias situações, não refletir as práticas reais de higiene bucal, sugerindo a possibilidade de viés de informação. Essa pode ser uma possível explicação para não ter sido identificada associação entre autopercepção da presença de cárie dentária e frequência de escovação.

Cumpre frisar que embora a autopercepção da saúde bucal tenha sido aferida, na presente investigação, como morbidade autorreferida (percepção da presença de cárie dentária), há na literatura outras concepções que abordam a autopercepção da saúde bucal sob diferentes enfoques, tais como avaliação da satisfação com a saúde bucal e percepção do estado da saúde bucal 3,8 e da necessidade de tratamento odontológico 1,6,41. Além disso, outros estudos trabalham a autopercepção da saúde bucal com enfoque no impacto que as condições de saúde bucal acarretam na qualidade de vida diária 7,35. Cabe também destacar que os resultados apresentados referem-se à validade da autopercepção da presença da cárie dentária obtida pela pergunta "Você acha que tem cárie dentária?”. Outras abordagens para aferir a autopercepção da cárie poderiam conduzir a diferentes resultados.

Em geral, a autoavaliação da saúde bucal é entendida como um indicador sintético, válido e útil do estado de saúde bucal 26. Portanto, os serviços de saúde precisam acessar as percepções de seus usuários, inclusive como forma de reduzir as iniquidades em saúde, ao considerá-las na organização dos serviços, definição de prioridades e atendimento das demandas. É imprescindível que os serviços de saúde atuem em prol da garantia de maior acesso a informações em saúde bucal, para que seus usuários se tornem cada vez mais conscientes de sua condição bucal e da relevância dos hábitos de autoexame e autocuidado, que devem ser estimulados especialmente entre a população adulta em razão da influência que exercem no comportamento de seus dependentes 18 . Vale ressaltar que, por sua natureza subjetiva e por sofrer influências contextuais, medidas de autopercepção devem ser geradas de forma específica para cada população, pois generalizações e comparações das medidas de autopercepção provenientes de diferentes populações precisam ser avaliadas com prudência.

O delineamento de corte transversal é uma limitação do estudo porque impede o estabelecimento de causa e efeito entre as variáveis, por não ser possível estabelecer uma relação temporal entre as associações observadas. Todavia, o plano amostral, a calibração dos examinadores, a coleta de dados registrada em computador de mão e a condução das análises tendo em conta a correção pelo efeito de desenho foram estratégias que garantiram validade e confiabilidade aos dados, minimizando a possibilidade do viés. Além disso, investigações com delineamento transversal são importantes, à medida que respaldam o desenvolvimento de ações de saúde por fornecerem dados imediatos da realidade do grupo observado e a geração de novas hipóteses.

\section{Conclusão}

Como teste diagnóstico, a autopercepção da presença de cárie dentária ainda apresenta limitações que inviabilizam seu uso em ampla escala, o que não significa que a mesma não tenha utilidade ou não possa ser usada em contextos específicos. A acurácia verificada foi limitada, com superestimação na percepção da presença de cárie dentária. Os relatos de sua presença são menos confiáveis do que de os sua ausência.

Constatou-se que a autopercepção da presença da cárie dentária esteve relacionada a contextos desfavoráveis: presença de sintomas e doença, insatisfação com a saúde bucal e geral, menor 
frequência de utilização dos serviços odontológicos, desinformação e hábitos de higiene inadequados. Ademais, a autopercepção da presença da cárie dentária se mostrou preditora da percepção da necessidade de tratamento odontológico, destacando sua importância na avaliação dos indivíduos para atendimento odontológico. Sendo assim, uso frequente dos serviços odontológicos e maior acesso a informações em saúde bucal apontam como caminho a ser perseguido para aumentar a validade e o poder preditivo da autopercepção da presença da cárie dentária.

\section{Colaboradores}

D. S. Haikal atuou na concepção do estudo, contribuiu na análise, interpretação dos dados e revisão crítica do artigo. L. L. Roberto colaborou na interpretação dos dados e redação do artigo. A. M. E. B. L. Martins atuou na concepção do estudo, trabalho de campo, processamento dos dados e revisão crítica do artigo. A. M. B. Paula atuou na revisão crítica do artigo. E. F. Ferreira atuou na interpretação dos dados e revisão crítica do artigo.

\section{Agradecimentos}

Os autores agradecem o apoio logístico da Unimontes e da Prefeitura Municipal de Montes Claros, o fomento da Fundação de Amparo à Pesquisa do Estado de Minas Gerais (FAPEMIG; EDT 3270/06) e o apoio financeiro recebido: D. S. Haikal é pesquisadora de produtividade da FAPEMIG. L. L. Roberto foi bolsista de mestrado da Capes. A. M. E. B. L. Martins é bolsista de pós-doutorado do CNPq, A. M. B. Paula e E. F. Ferreira são pesquisadores de produtividade do CNPq. 


\section{Referências}

1. Gilbert GH, Heft MW, Duncan RP, Ringelberg ML. Perceived need for dental care in dentate older adults. Int Dent J 1994; 44:145-52.

2. Srisilapanan P, Sheiham A. Assessing the difference between sociodental and normative approaches to assessing prosthetic dental tratament needs in dentate older people. Gerodontology 2001; 18:25-34.

3. Locker D, Miller Y. Subjectively reported oral health status in an adult population. Community Dent Oral Epidemiol 1994; 22:425-30.

4. Bedos C, Brodeur JM, Levine A, Richard L, Boucheron L, Mereus W. Perception of dental illness among persons receiving public assistance in Montreal. Am J Public Health 2005; 95:1340-4.

5. Lundegren N, Axtelius B, Hakansson J, Akerman S. Dental treatment need among 20 to 25-year-old Swedes: discrepancy between subjective and objective need. Acta Odontol Scand 2004; 62:91-6.

6. Slaughter A, Taylor L. Perceptions of dental care need among African-American elders: implications for health promotion. Spec Care Dentist 2005; 25:158-63.

7. Blaizot A, Monsarrat P, Constantin A, Vergnes JN, Grado GF, Nabet C, et al. Oral healthrelated quality of life among outpatients with rheumatoid arthritis. Int Dent J 2013; 63:14553.

8. Haikal DS, De-Paula AMB, Martins AMEBL, Moreira AN, Ferreira EF. Autopercepção da saúde bucal e impacto na qualidade de vida do idoso: uma abordagem quanti-qualitativa. Ciênc Saúde Coletiva 2011; 16:3317-29.

9. Soares GB, Batista RM, Zandonade E, Oliveira AE. Association of self-perception of oral health with clinical oral parameters. Rev Bras Odontol 2011; 68:268-73.

10. Gilbert AD, Nuttall NM. Self-reporting of periodontal health status. Br Dent J 1999; 186:241-4.

11. Pinelli C, Loffredo LCM. Reproducibility and validity of self-perceived oral health conditions. Clin Oral Invest 2007; 11:431-7.

12. World Health Organization. Oral health surveys: basic methods. 4th Ed. Geneva: World Health Organization; 1997.
13. World Health Organization. Calibration of examiners for oral health epidemiological surveys. Geneva: World Health Organization; 1993.

14. Narvai PC. Collective oral health: ways from sanitary dentistry to buccality. Rev Saúde Pública 2006; 40:141-7.

15. Selwitz RH, Ismail AI, Pitts NB. Dental caries. Lancet 2007; 369:51-9.

16. Ministério da Saúde. Projeto SB Brasil 2010: resultados principais. Brasília: Ministério da Saúde; 2011

17. Jamieson LM, Thomson WM. The Dental Neglect and Dental Indifference scales compared. Community Dent Oral Epidemiol 2002; 30:168-75

18. Pinto VG. Saúde bucal coletiva. 4ạ Ed. São Paulo: Editora Santos; 2000.

19. Coordenação Nacional de Saúde Bucal, Departamento de Atenção Básica, Secretaria de Atenção à Saúde, Ministério da Saúde. Projeto SB Brasil 2003. Condições de saúde bucal da população brasileira 2002-2003: resultados principais. Brasília: Ministério da Saúde; 2004

20. Cicchetti DV, Volkmar F, Sparrow SS, Cohen D, Fermanian J, Rourke BP. Assessing the reliability of clinical scales when the data have both nominal and ordinal features: proposed guidelines for neuropsychological assessments. J Clin Exp Neuropsychol 1992; 14:67386.

21. Cascaes AM, Peres KG, Peres MA. Periodontal disease is associated with poor self-rated oral health among Brazilian adults. J Clin Periodontol 2009; 36:25-33.

22. Pitiphat W, Garcia RI, Douglass CW, Joshipura KJ. Validation of self-reported oral health measures. J Public Health Dent 2002; 62:122-8.

23. Green LW, Kreuter MW. Health promotion planning: an educational and environmental approach. Mountain View: Mayfield Publishing Company; 1991.

24. Zanardo A, Rego MA. Occlusal caries diagnosis in permanent teeth: an in vitro study. Ciênc Odontol Bras 2003; 6:50-7. 
25. Gordis L. Epidemiologia. 4a Ed. Rio de Janeiro: Revinter; 2010.

26. Kojima A, Ekuni D, Mizutani S, Furuta M, Irie $\mathrm{K}$, Azuma T, et al. Relationships between self-rated oral health, subjective symptoms, oral health behavior and clinical conditions in Japanese university students: a cross-sectional survey at Okayama University. BMC Oral Health 2013; 13:62.

27. Matos DL, Lima-Costa MF. Auto-avaliação da saúde bucal entre adultos e idosos residentes na Região Sudeste: resultados do Projeto SB-Brasil, 2003. Cad Saúde Pública 2006; 22:1699-707.

28. Martins AMEBL, Barreto SM, Silveira MF, Santa-Rosa TTA, Pereira RD. Autopercepção da saúde bucal entre idosos brasileiros. Rev Saúde Pública 2010; 44:912-22.

29. Gondim CG, Moura WVB, Lucena RGR, Silva BR, Vasconcelos HM, Aguiar ASW. Saúde bucal de pacientes internados em hospital de emergência. Arq Odontol 2012; 48:270-9.

30. Sistani MMN, Yazdani R, Virtanen J, Pakdaman A, Murtomaa H. Determinants of oral health: does oral health literacy matter? ISRN Dentistry 2013; 2013:249591

31. Martins AMEBL, Jones KM, Souza JGS, Pordeus IA. Associação entre impactos funcionais e psicossociais das disordens bucais e qualidade de vida entrre idosos. Ciênc Saúde Coletiva 2014; 19:3461-78.

32. Moura C, Gusmão ES, Santillo PMH, Soares RSC, Cimões R. Autoavaliação da saúde bucal e fatores associados entre adultos em áreas de assentamento rural, Estado de Pernambuco, Brasil. Cad Saúde Pública 2014; 30:611-22.

33. Andrade FB, Lebrão ML, Santos JLF, Duarte YAO, Teixeira DSC. Fatores associados à autopercepção de saúde bucal ruim entre idosos não institucionalizados do Município de São Paulo, Brasil. Cad Saúde Pública 2012; 28:1965-75
34. Mathias RE, Atchison KA, Lubben JE, De Jong F, Schweitzer SO. Factors affecting self-ratings of oral health. J Public Health Dent 1995; 55:197-204.

35. Slade GD, Spencer AJ. Development and evaluation of the Oral Health Impact Profile. Community Dent Health 1994; 11:3-11.

36. Gilbert GH, Duncan RP, Shelton BJ. Social determinants of tooth loss. Health Serv Res 2003; 38:1843-62.

37. Haikal DS, Martins AMEBL, Aguiar PHS, Silveira MF, Paula AMB, Ferreira EF. O acesso à informação sobre higiene bucal e as perdas dentárias por cárie entre adultos. Ciênc Saúde Coletiva 2014; 19:287-300.

38. Martins AMEBL, Barreto SM, Santos-Neto PE, Sá MAB, Souza JGS, Haikal DS, et al. Maior acesso à informação sobre como prevenir o câncer bucal entre idosos assistidos na atenção primária à saúde. Ciênc Saúde Coletiva $2015 ; 20: 2239-53$.

39. Pinelli C, Turrioni APS, Loffredo LCM. Autopercepção em higiene bucal de adultos: reprodutibilidade e validade. Rev Odontol UNESP 2008; 37:163-9.

40. Baldani MH, Brito WH, Lawder JAC, Mendes YBE, Silva FFM, Antunes JLF. Determinantes individuais da utilização de serviços odontológicos por adultos e idosos de baixa renda. Rev Bras Epidemiol 2010; 13:150-62.

41. Moreira RS, Nico LS, Sousa MLR. Fatores associados à necessidade subjetiva de tratamento odontológico em idosos brasileiro. Cad Saúde Pública 2009; 25:2661-71. 


\section{Abstract}

This study aimed to analyze the validity of selfperceived dental caries and associated factors in a sample of 795 adults (35-44 years). The dependent variable was self-perceived dental caries, and the independent variables were combined in blocks. Three logistic models were performed: (1) all adults; (2) adults with a formal diagnosis of caries; and (3) adults without such caries. Selfperceived dental caries showed $77.7 \%$ sensitivity, $58 \%$ specificity, $65 \%$ accuracy, $52 \%$ positive predictive value, and $81 \%$ negative predictive value. In Model 1, self-perceived dental caries was associated with time of use of dental services, access to information, flossing, formal diagnosis of caries, self-perceived need for treatment, toothache, and dissatisfaction with oral health and general health. In Model 2, self-perceived dental caries was associated with time of use of dental services, self-perceived need for treatment, and dissatisfaction with oral health and general health. In Model 3 , self-perceived dental caries was associated with time of use of dental services, access to information, flossing, self-perceived need for treatment, and dissatisfaction with oral health. Self-perceived dental caries showed limited utility as a diagnostic method.

Self Concept; Dental Caries; Oral Health

\section{Resumen}

Se tuvo como objetivo investigar la validez de la autopercepción de la presencia de la caries dental y factores asociados, mediante un estudio con 795 adultos (35-44 años). La variable dependiente fue autopercepción de la presencia de la caries dental, las independientes se reunieron en bloques. Se realizaron tres modelos logísticos: (1) todos los adultos; (2) adultos con lesiones de caries conforme a la norma; y (3) adultos sin tales lesiones. La autopercepción de la presencia de la caries dental presentó sensibilidad de un 77,7\%, especificidad de un 58\%, precisión de un $65 \%$, valor predictivo positivo de un $52 \%$ y valor predictivo negativo de un $81 \%$. En el Modelo 1, la autopercepción de la presencia de la caries dental se asoció al tiempo de uso de los servicios odontológicos, acceso a la información, uso de hilo dental, lesiones conforme a la norma, percepción de la necesidad de tratamiento, dolor, insatisfacción con la salud bucal y general. En el Modelo 2, la autopercepción de la presencia de la caries dental se asoció al tiempo de uso de los servicios odontológicos, percepción de la necesidad de tratamiento e insatisfacción con la salud bucal y general. En el Modelo 3, la autopercepción de la presencia de la caries dental se asoció al tiempo de uso de los servicios odontológicos, acceso a la información, percepción de la necesidad de tratamiento, insatisfacción con la salud bucal y uso de hilo dental. La autopercepción de la presencia de la caries dental mostró utilidad limitada como método de diagnóstico.

Autoimagen; Caries Dental; Salud Bucal
Recebido em 01/Abr/2016

Versão final reapresentada em 21/Jul/2016

Aprovado em 22/Set/2016 